\title{
Zwischen der Bagatellisierung sexueller Gewalt und drakonischen Strafen
}

\author{
Zum Umgang mit sexuellem Kindesmissbrauch im Nationalsozialismus
}

\author{
Dagmar Lieske
}

\section{Einführung}

Im Dezember 1967 wandte sich Ludwig Rosenberg1, der Vorsitzende des Deutschen Gewerkschaftsbundes (DGB), mit folgender Bitte an den damaligen Bundesjustizminister Gustav Heinemann: Dieser möge ihm Auskunft darüber erteilen, wie viele Strafverfahren wegen sexuellen Kindesmissbrauchs in der Zeit des Nationalsozialismus geführt wurden. ${ }^{2}$ Aktuelle Taten, so Rosenbergs Vermutung, würden „in der Öffentlichkeit und auch in Arbeiterkreisen“ die Position begünstigen, so etwas habe es „unter den Nazis nie gegeben“. Gemeint waren hier vermutlich u.a. die medial intensiv behandelten Verbrechen von Jürgen Bartsch, der im Juni 1966 festgenommen und im November 1967 wegen Mordes an vier Jungen vor dem Landgericht Wuppertal angeklagt wurde. ${ }^{3}$

Rosenberg konstatierte nicht nur ein verbreitetes Unwissen über sexuelle Gewalt im Nationalsozialismus, sondern befürchtete auch, dieses helfe der rechtsextremen NPD dabei, die „Stimmungsmache zu stärken“. In der Tat entsprach die offensichtlich auch in den 196oer Jahren noch verbreitete Vorstellung eines nahezu kriminalitätsfreien „Dritten Reiches“ dem Selbstbild

1 Ludwig Rosenberg (1903-1977) war schon in der Weimarer Republik aktiver Gewerkschafter. Aufgewachsen in einer gutbürgerlichen jüdischen Familie floh er 1933 vor den Nationalsozialisten nach London, pflegte Kontakte zu Gewerkschafter:innen im Exil und war für das britische Außenministerium tätig. Seine Mutter sowie weitere Angehörige wurden im Vernichtungslager Auschwitz-Birkenau ermordet. Nach 1945 kehrte Rosenberg nach Deutschland zurück. Er war am Aufbau des DGB beteiligt und wurde ab Oktober 1962 dessen Vorsitzender. Rosenberg verstarb 1977 in Düsseldorf, vgl. zu seiner Biografie Frank Ahland, Bürger und Gewerkschafter Ludwig Rosenberg 1903 bis 1977. Eine Biographie. Essen 2016.

2 Alle folgenden Zitate aus Bundesarchiv (BArch), B 141 63807. Den Hinweis auf dieses Dokument verdanke ich Jan-Henrik Friedrichs.

3 Vgl. Kerstin Brückweh, Ein Sexualstraftäter in Medien, Gesellschaft und Strafvollzug. Das Beispiel Jürgen Bartsch, in: Ansgar Weißer (Hrsg.), Psychiatrie - Geschichte - Gesellschaft: Das Beispiel Eickelborn im 20. Jahrhundert. Bonn 2009, 125-144; dies., Fantasies of Violence. German citizens expressing their concepts of violence and ideas about democracy in letters referring to the case of the serial killer Jürgen Bartsch (1966-1971), in: Crime, History and Societies 10(2), 2006, 2-25. 
der Nationalsozialisten. ${ }^{4}$ Nach der Machtübernahme wurde der angebliche Erfolg neuer kriminalpolitischer Maßnahmen entsprechend propagandistisch begleitet. Darunter fiel auch die Zwangskastration von "Sittlichkeitsverbrechern“ (u.a. „Kinderschändern“5), mit der sich der spätere niedersächsische Justizminister Hans Puvogel (CDU) in seiner 1937 erschienenen Dissertationsschrift befasste. Der „Sittlichkeitsverbrecher", so Puvogel, unterliege „trotz schwerster Strafen stets wieder seinem unnatürlichen Drange zum Verbrechen." Seine „Unschädlichmachung“ sei daher „im Interesse eines wirksamen Schutzes der Volksgemeinschaft unbedingt erforderlich.“ ${ }^{\text {}}$

Hier klingt bereits an, was für die Debatte und Verfolgungspraxis von sexueller Gewalt im Nationalsozialismus zentral war: Nicht der Schutz des Individuums stand im Vordergrund, sondern vielmehr der einer (imaginierten) „Volkgemeinschaft“7. Die „Entmannung“ von „Kinderschändern“ war zwar schon in der Weimarer Republik wiederholt gefordert worden, ${ }^{8}$ avancierte aber erst mit der Einführung des „Gesetzes gegen gefährliche Gewohnheitsverbrecher und über Maßregeln der Sicherung und Besserung" (kurz: Gewohnheitsverbrechergesetz) im November 1933 zu einer gängigen kriminalpolitischen Maßnahme. ${ }^{9}$ Nach dem Ende des Zweiten Weltkrieges setzte der Alliierte Kontrollrat die Möglichkeit einer richterlichen Anordnung von Zwangskastrationen aus. Körperliche Eingriffe blieben aber als strafpräventives

4 Vgl. dazu Michael Hepp, „Bei Adolf wäre das nicht passiert“? Die Kriminalstatistik widerlegt eine zählebige Legende, in: Zeitschrift für Rechtspolitik 32(6), 1999, 253-16o.

5 Der Begriff „Kinderschänder“ wird hier als zeitgenössischer Begriff zitiert und in Anführungszeichen gesetzt, ist aus meiner Sicht aber höchst problematisch, weil er nicht nur Personen auf ein Delikt reduziert, sondern zudem die Betroffenen herabsetzt, indem sie als „geschändet“ und damit als „beschädigt“" gelten, vgl. dazu auch Brigitte Kerchner, Körperpolitik. Die Konstruktion des „Kinderschänders“ in der Zwischenkriegszeit, in: Wolfgang Hardtwig (Hrsg.), Politische Kulturgeschichte der Zwischenkriegszeit 1918-1939. Göttingen 2005, 241-279.

6 Hans Puvogel, Die leitenden Grundgedanken bei der Entmannung gefährlicher Sittlichkeitsverbrecher. Düsseldorf 1937, 26. Hans Puvogel (1911-1999) promovierte mit dieser Schrift 1936 an der Georg-August-Universität in Göttingen. Seine im Mai 1976 erlangte Position als niedersächsischer Justizminister musste er 1978 nach Bekanntwerden der Dissertationsschrift wieder aufgeben. Er hat sich von den dort getätigten Äußerungen nie distanziert, vgl. dazu Susanne zur Nieden, Homophobie und Staatsräson, in: dies. (Hrsg.), Homosexualität und Staatsräson. Männlichkeit, Homophobie und Politik in Deutschland 1900-1945. Frankfurt a. M. 2005, 17-52, hier 42.

7 Vgl. zum Begriff der „Volksgemeinschaft“ Michael Wildt, „Volksgemeinschaft“, in: DocupediaZeitgeschichte, 3.6.2014 (Version 1.o), einzusehen unter https://docupedia.de/zg/Volksgemeinschaft, zuletzt aufgerufen am 19.11.2020.

8 Vgl. z.B. bei Borwin Himmelreich, Die Kinderschändung. Leipzig 1932.

9 Vgl. zu dem Gesetz Christian Müller, Das Gewohnheitsverbrechergesetz vom 24. November 1933. Kriminalpolitik als Rassenpolitik. Baden-Baden 1997. 
Mittel weiterhin wichtiger Bestandteil kriminalpolitischer und sexualwissenschaftlicher Debatten. Das 1969 implementierte "Gesetz über die freiwillige Kastration und andere Behandlungsmethoden" erlaubte schließlich auch in der Bundesrepublik die (allerdings freiwillige) Kastration von Delinquenten mit „abnormem Geschlechtstrieb“ ${ }^{10}$ Darunter fielen auch Männer, die Kinder und Jugendliche sexuell missbraucht hatten - wie der zuvor erwähnte Jürgen Bartsch, der 1976 in Folge einer freiwilligen Kastration im Landeskrankenhaus Eickelborn verstarb.

An dieser Stelle zeigt sich einmal mehr, dass von einem „Bruch“ oder gar einer "Stunde Null“11 mit dem Ende des Zweiten Weltkrieges nicht die Rede sein kann. Auch hat die von Rosenberg für die 196oer Jahre beobachtete Instrumentalisierung von Fällen sexueller Gewalt durch neurechte und neonazistische Strömungen bis heute nicht an Aktualität eingebüßt, wie verschiedene Studien belegen. ${ }^{12}$

\section{Desiderate}

Rosenbergs Ziel, das Ausmaß des sexuellen Kindesmissbrauchs für die Zeit des Nationalsozialismus sichtbar zu machen, scheint weiterhin unerreicht handelt es sich doch um ein Thema, das bislang weder in der Öffentlichkeit noch in der Forschung viel Beachtung gefunden hat. ${ }^{13}$ Die wenigen Studien, die

10 Annelie Ramsbrock, Das verlorene Geschlecht. Zur Kastration von Sexualstraftätern seit 1945, in: Themenportal Europäische Geschichte, 2016, einzusehen unter www.europa. clio-online.de/essay/id/artikel-3142, zuletzt aufgerufen am 19.11.2020.

11 Steffi Hobuß, Mythos „Stunde Null“, in: Torben Fischer/Matthias N. Lorenz (Hrsg.), Lexikon der ,Vergangenheitsbewältigung“ in Deutschland. Debatten- und Diskursgeschichte des Nationalsozialismus nach 1945. 3. Aufl. Bielefeld 2015, 45.

12 Robert Claus/Fabian Virchow, The Far Right's Ideological Constructions of ,Deviant' Male Sexualities, in: Michaela Köttig/Renate Bitzan/Andrea Petö (Hrsg.), Gender and Far Right Politics in Europe. London 2017, 305-319; Imke Schmincke, Das Kind als Chiffre politischer Auseinandersetzung am Beispiel neuer konservativer Protestbewegungen in Frankreich und Deutschland, in: Sabine Hark/Paula-Irene Villa (Hrsg.), AntiGenderismus: Sexualität und Geschlecht als Schauplätze aktueller politischer Auseinandersetzungen. Bielefeld 2015, 93-107.

13 Im Rahmen ihrer eigenen Forschung hat die Autorin dieses Beitrags dazu einzelne Beiträge vorgelegt, vgl. Dagmar Lieske, Von „Gemeingefährlichen“, „Sittlichkeitsverbrechern“ und „Geschändeten“. Die Verfolgung von sexuellem Kindesmissbrauch im Nationalsozialismus, in: Stefan Grüner/Markus Raasch (Hrsg.), Zucht und Ordnung. Gewalt gegen Kinder in historischer Perspektive. Berlin 2019, 403-431; dies., Zwischen repressivem Maßnahmenstaat und der Bagatellisierung sexueller Gewalt - Zur strafrechtlichen Verfolgung von Kindesmissbrauch im Nationalsozialismus, in: Sexuologie 25(3-4), 2018, 193-199. 
Kindesmissbrauch im Nationalsozialismus thematisieren, fokussieren auf die Zuspitzung biopolitischer Kriminalpolitik und behandeln spezifische Aspekte wie die Zwangskastrationen und/oder die Einweisung von "Sittlichkeitsverbrechern" in Konzentrationslager. ${ }^{14}$ Einzelne Impulse kommen auch aus der Forschung über die Sanktionierung gleichgeschlechtlicher Beziehungen. ${ }^{15}$ Während die Rolle der Justiz und „abweichendes Verhalten“ in der Forschung intensiv behandelt worden sind, wurde die "reguläre" Strafrechtpraxis im Nationalsozialismus bislang nur selten untersucht. ${ }^{16}$ Der Historiker Dirk Blasius hat sich etwa Ehescheidungen gewidmet und kommt zu dem Schluss, die Jahre zwischen 1933 und 1945 seien "nicht ausschließlich als Extremjahre der deutschen Rechtsentwicklung zu sehen“, denn: „Hinter den Extrembefunden, die in der Zeit des Nationalsozialismus begegnen, verlaufen rechtsgeschichtliche Kontinuitätslinien, die auf den Bildungsprozeß des Rechts in der Moderne, seine Einlagerung in den Entstehungs- und Entfaltungsvorgang der modernen, bürgerlichen Gesellschaft verweisen. ${ }^{17}$

Daran anknüpfend liegt die Herausforderung darin, die nationalsozialistische Spezifik herauszufiltern, ohne dabei Kontinuitäten zu ignorieren ${ }^{18}$ und Widersprüchlichkeiten zu übergehen, die die Herrschaftspraxis in den Jahren zwischen 1933 und 1945 in vielen Bereichen durchzogen. Kann tatsächlich davon ausgegangen werden, dass sexuelle Gewalt gegen Kinder und Jugendliche im Nationalsozialismus grundsätzlich intensiver

14 Vgl. z.B. Greg Eghigian, The Corrigible and the Incorrigible. Science, Medicine and the Convict in Twentieth-Century Germany. Michigan 2015; Silke Schneider, Sexualdelikte im Nationalsozialismus. Opfer- und Täterbilder, in: Christine Künzel (Hrsg.), Unzucht Notzucht - Vergewaltigung. Definitionen und Deutungen sexueller Gewalt von der Aufklärung bis heute. Frankfurt a. M./New York 2003, 165-185.

15 Albert Knoll, „Ein System steht zur Anklage“. Die Verfahren gegen Geistliche wegen Sittlichkeitsvergehen im Nationalsozialismus und die Reaktion der Kirche, in: Werkstatt Schwule Theologie 4, 1999, 276-285; Andreas Sternweiler, Und alles wegen der Jungs: Pfadfinderführer und KZ-Häftling: Heinz Dörmer. Hamburg 1994; Hans Günter Hockerts, Die Sittlichkeitsprozesse gegen katholische Ordensangehörige und Priester 1936/37. Eine Studie zur nationalsozialistischen Herrschaftstechnik und zum Kirchenkampf. Mainz 1971.

16 Die Fülle an Literatur über die Justiz im Nationalsozialismus ist inzwischen nahezu unüberschaubar; die meisten Studien untersuchen einzelne Aspekte oder lokale Gerichte. Für einen Überblick zur Rolle der Justiz vgl. Redaktion Kritische Justiz (Hrsg.), Der Unrechtsstaat. Recht und Justiz im Nationalsozialismus. Frankfurt a. M. 1979; Lothar Gruchmann, Justiz im Dritten Reich, 1933-1940, Anpassung und Unterwerfung in der Ära Gürtner. Oldenburg 2001.

17 Dirk Blasius, Ehescheidung in Deutschland im 19. und 20. Jahrhundert. Frankfurt a. M. 1992, 188.

$18 \mathrm{Zu}$ den Kontinuitäten im Hinblick auf Bilder von Betroffenen und Täter:innen vgl. Kerchner, Körperpolitik. 
geahndet wurde, als etwa in der Weimarer Republik, wie es beispielsweise die Inszenierung des Staates als "Sittenwächter" im Kontext der „Priesterprozesse“ in den Jahren 1936/37 Glauben machen will?19 Basierend auf der Analyse von Strafverfahren, die nach $\S 176$ Absatz 3 zwischen Februar 1933 und April 1945 vor dem Landgericht Berlin geführt wurden, wird dieser Frage im Folgenden nachgegangen. ${ }^{20}$ Der $\S 176$ Absatz 3, der mit der Einführung des Reichsstrafgesetzbuches 1872 in Kraft trat, stellte "unzüchtige Handlungen mit bzw. an Personen unter 14 Jahren" unter Strafe und blieb im Nationalsozialismus unverändert. ${ }^{21}$ Er sah (und sieht in mehrfach reformierter Form) ein Höchststrafmaß von zehn Jahren Zuchthaus (heute Gefängnis) sowie ein Mindeststrafmaß von sechs Monaten Gefängnis vor. ${ }^{22}$

\section{Quellen und Quellenanalyse}

Sexuelle Gewalt gegen Kinder und Jugendliche lässt sich in allen Phasen der neueren deutschen Geschichte bis hinein in die Gegenwart finden und beschreiben. Bei einer vergleichenden Betrachtung der verschiedenen historischen Kontexte stellen sich schnell folgende Fragen: Was galt in den jeweiligen Gesellschaften als sexuell und folglich auch als sexuelle Gewalt? Wie waren Kindheit und Jugend definiert und welche Mittel standen bei der Ahndung von sexuellem Kindesmissbrauch zur Verfügung? Auf welche Weise wurden Betroffene und Täter:innen attribuiert? Wie die Juristin Garonne Bezjak resümiert, kann sexueller Missbrauch je nach Rechtslage „etwa als normale Erscheinung, als moralisch anstößig oder als sanktionswürdig erlebt werden“. ${ }^{23}$ Zudem kommt es nicht nur auf die Analyse des jeweils geltenden Rechts an, sondern auch auf dessen Anwendung. Nicht zuletzt findet Rechtsprechung immer vor dem Hintergrund gesellschaftlicher Debatten und Aushandlungsprozesse statt. Während die Rekonstruktion historischer Diskurse noch möglich ist, bleibt der Zugang zum sogenannten Dunkelfeld, also zu Fällen, in denen es nicht zur Anzeige bzw. Strafverfolgung gekommen

\footnotetext{
19 Vgl. dazu den Beitrag von Hans Günter Hockerts in diesem Band.

20 Es handelt sich um den Bestand Strafakten des Berliner Landgerichts im Landesarchiv Berlin (LAB), A Rep. 358-o2.

21 Garonne Bezjak, Grundlagen und Probleme des Straftatbestandes des sexuellen Missbrauchs von Kindern gemäß $§ 176$ StGB. Berlin 2015. Der Paragraf besteht (mit verschiedenen Erneuerungen) bis heute.

$22 \S 176$ StGB, einzusehen unter https://dejure.org/gesetze/StGB/176.html, zuletzt aufgerufen am 19.11.2020.

23 Bezjak, Grundlagen, 17.
} 
ist, insbesondere dann versperrt, je weiter wir in die Vergangenheit zurückblicken. Die Geschichte sexueller Gewalt kann deshalb häufig nur anhand von juristischen und/oder institutionellen Dokumenten geschrieben werden. Aufgrund dieser Verengung ist ein kritischer, diskursanalytischer Blick auf die vorhandenen Quellen hilfreich, der den jeweiligen gesellschaftlichen Kontext berücksichtigt. Dies gilt etwa für die Justizakten aus der Zeit des Nationalsozialismus, in denen sich stigmatisierende Zuschreibungen sowohl über Betroffene als auch Täter:innen finden, die den zeitgenössischen Diskurs widerspiegeln.

Anhand von Verhandlungen vor dem Berliner Landgericht wird im Folgenden aufgezeigt, dass es zwischen 1933 und 1945 nicht nur einen Umgang mit Fällen von sexuellem Kindesmissbrauch gab - vielmehr changierte die nationalsozialistische Strafrechtspraxis zwischen einer auch schon vor $1933 \mathrm{zu}$ beobachtenden Bagatellisierung sexueller Gewalttaten und der Verhängung drakonischer Strafen bis hin zur Vernichtung einzelner Personen, die als „Kinderschänder" galten.

\section{Nationalsozialistische Kriminalpolitik und Sexualstrafrecht}

Für die Quellenanalyse ist eine sorgfältige Betrachtung des historischen Kontextes wichtig, gerade wenn Kontinuitäten und Brüche untersucht werden sollen. Zu nennen wäre hier zunächst die konkrete Ausgestaltung und die Anwendung des Sexualstrafrechts zwischen 1933 und 1945 sowie die begleitenden Fachdebatten. Das geltende Sexualstrafrecht flankierten zudem ab Ende 1933 neue kriminalpolitische Instrumente, die einbezogen werden müssen. ${ }^{24}$ Ferner ist die Phase von Bedeutung, in der sich das nationalsozialistische Regime jeweils befand: Konsolidierung und Machtübernahme; Aufrüstung; Zweiter Weltkrieg und die Endphase des Regimes sind wichtige Marker für den Verlauf der nationalsozialistischen Herrschaft, die auch die Praxis der Strafverfolgung beeinflusst haben. All dies geschah vor dem Hintergrund einer spezifischen Konstruktion der Gesellschaft zur "Volksgemeinschaft“,

24 Julia Hörath, „Asoziale“ und „Berufsverbrecher" in den Konzentrationslagern 1933 bis 1938. Göttingen 2017; Dagmar Lieske, Unbequeme Opfer? „Berufsverbrecher“ als Häftlinge im KZ Sachsenhausen. Berlin 2016; Thomas Roth, Verbrechensbekämpfung und soziale Ausgrenzung im nationalsozialistischen Köln: Kriminalpolizei, Strafjustiz und abweichendes Verhalten zwischen Machtübernahme und Kriegsende. Köln 2010; Patrick Wagner, Volksgemeinschaft ohne Verbrecher. Konzeptionen und Praxis der Kriminalpolizei in der Zeit der Weimarer Republik und des Nationalsozialismus. Hamburg 1996. 
für die Antisemitismus, Rassismus, Eugenik und Anti-Feminismus ${ }^{25}$ zentral waren und in die Urteilspraxis einflossen. Hier stellt sich auch die Frage nach einer Reproduktion von genderspezifischen Zuschreibungen auf Täter:innen ${ }^{26}$ und Betroffene in den Gerichtsverfahren. ${ }^{27}$

Da sich auf der Ebene des Sexualstrafrechts nach 1933 nichts änderte und die bereits im Kaiserreich etablierten $\S 174$ bis 184 StGB unverändert blieben, ist Silke Schneider insofern zuzustimmen, dass (abgesehen vom $\S 175^{28}$ ) für das Sexualstrafrecht im Nationalsozialismus weniger eine „Verschärfung der materiellen Normen“ kennzeichnend war, „als die radikale Verfolgung abweichenden Verhaltens“. ${ }^{29}$

Im Zweiten Weltkrieg traten schließlich Verschärfungen in Kraft. So war ab 1941 die Verhängung von Todesurteilen bei einer Bestrafung nach $\S \S 176-178$ StGB möglich. Eine weitere Änderung vollzog sich in Bezug auf $\S 174$ StGB, der "Unzucht“ an bzw. mit minderjährigen "Schutzbefohlenen“ unter Strafe stellte und ein Höchstmaß von fünf Jahren Zuchthaus vorsah. ${ }^{30}$ Interessanterweise hatten leibliche Kinder und Jugendliche bis zu diesem Zeitpunkt nicht als „Schutzbefohlene“ im Sinne des § 174 StGB gegolten. Möglicherweise liegt dies auch in der Ignoranz gegenüber der Familie als Tatort begründet: Als

25 Vgl. Elke Frietsch/Christina Herkommer (Hrsg.), Nationalsozialismus und Geschlecht. Zur Politisierung und Ästhetisierung von Körper, „Rasse“ und Sexualität im „Dritten Reich“ und nach 1945. Bielefeld 2009; Klaus Latzel/Elissa Mailänder/Franka Maubach (Hrsg.), Geschlechterbeziehungen und „Volksgemeinschaft“. Göttingen 2018.

26 Unter den wegen sexuellem Kindesmissbrauch zwischen 1933 und 1945 vor dem Berliner Landgericht angeklagten Personen befanden sich auch einige wenige Frauen, unter den Betroffenen sowohl Mädchen als auch Jungen. Da der gesamte Bestand von etwa 7oo Fallakten noch nicht ausgewertet wurde, kann die genaue Anzahl hier nicht angeben werden. Vgl. Michael Löffelsender, Frauen vor Gericht. Geschlechtsspezifische Zuschreibungspraktiken in der nationalsozialistischen Strafrechtsprechung im Krieg, in: Gaby Temme/ Christine Künzel (Hrsg.), Hat Strafrecht ein Geschlecht? Zur Deutung und Bedeutung der Kategorie Geschlecht in strafrechtlichen Diskursen vom 18. Jahrhundert bis heute. Bielefeld 2010, 195-212; Thomas Roth, „Gestrauchelte Frauen“ und „unverbesserliche Weibspersonen“: Zum Stellenwert der Kategorie Geschlecht in der nationalsozialistischen Strafrechtspflege, in: Frietsch/Herkommer, Nationalsozialismus und Geschlecht, 109-140. Der $§ 175$, der seit 1872 gleichgeschlechtliche Handlungen unter Strafe stellte, wurde 1935 dahingehend verschärft, dass er nun jede Form von Kontakten betraf, die als „wollüstig“ ausgelegt werden konnten (darunter z.B. auch Blicke), gleichzeitig wurde die Höchststrafe von sechs Monaten auf fünf Jahre Gefängnis angehoben und der § 175a für „schwere Fälle" eingeführt, vgl. für einen Überblick Freunde eines Schwulen Museums in Berlin e.V. (Hrsg.), Die Geschichte des § 175. Strafrecht gegen Homosexuelle. Katalog zur Ausstellung in Berlin und Frankfurt am Main 199o. Berlin 199o.

29 Schneider, Sexualdelikte im Nationalsozialismus, 168.

$30 § 174$ StGB, einzusehen unter https://dejure.org/gesetze/StGB/174.html, zuletzt aufgerufen am 19.11.2020. 
Verursacher von Kindesmissbrauch wurden (und werden häufig auch heute noch) in erster Linie „fremde“ Täter:innen wahrgenommen. Erst ab Juni 1943 fasste das Strafrecht "Betreuung“ allgemeiner und integrierte nun auch leibliche „Schutzbefohlene“. An dieser Stelle wäre zu diskutieren, warum ausgerechnet der nationalsozialistische Staat den § 174 StGB auf den familiären Nahbereich ausdehnte. Möglicherweise zielte die Gesetzesänderung auf eine zunehmende Kontrolle von Familie und Privatheit im Nationalsozialismus ab und/oder ist als ein Aspekt der allgemeinen Strafrechtsverschärfungen im Zweiten Weltkrieg zu interpretieren.

\section{Recht und Rechtsprechung im Nationalsozialismus - Quellenbeispiele}

Für die Einschätzung des juristischen Umgangs mit sexuellem Kindesmissbrauch nach 1933 ist ein Blick auf die Auslegung des geltenden Rechts hilfreich. Anhand von einzelnen Quellenbeispielen wird im Folgenden aufgezeigt, welchen Einfluss die Bewertung der Täter:innenperson auf das Strafmaß hatte. Relevant waren dabei sowohl das Alter, die soziale Herkunft als auch das Geschlecht der Angeklagten.

Einer der „Tätertypen“, über den seit 1933 auch im Kontext von Kindesmissbrauch intensiv debattiert wurde, war der „Exhibitionist“. In der Monatsschrift für Kriminalpsychologie und Strafrechtsreform, einem führenden kriminalpolitischen Fachorgan, ${ }^{31}$ entspann sich eine Debatte darüber, ob Fälle von Exhibitionismus vor Kindern und Jugendlichen nach § 176 Abs. 3 StGB und nicht mehr (wie in der Weimarer Republik üblich) nach § 183 StGB (Exhibitionismus) $^{32}$ oder $\S 185$ StGB (Beleidigung) ${ }^{33}$ verhandelt werden sollten. Diese Entscheidung konnte erhebliche Auswirkungen auf das Strafmaß haben, denn die $\S \S 183$ und 185 StGB sahen eine maximale Haftstrafe von zwei Jahren Gefängnis vor, während Vergehen gegen den $\S 176$ StGB mit bis zu zehn Jahren Zuchthaus sanktioniert werden konnten.

31 Wie eine erste Auswertung der Jahrgänge 1933 bis 1945 ergeben hat, wurden neben Exhibitionismus in der Zeitschrift im Zusammenhang mit sexuellem Kindesmissbrauch in diesem Zeitraum überwiegend die Themen „Blutschande“/,Inzest“, Homosexualität und Kastrationen diskutiert.

$32 \S 183$ StGB, einzusehen unter https://dejure.org/gesetze/StGB/183.html, zuletzt aufgerufen am 19.11.2020.

$33 \S 185$ StGB, einzusehen unter https://dejure.org/gesetze/StGB/185.html, zuletzt aufgerufen am 19.11.2020. 
Für Kontroversen sorgte im Nationalsozialismus die Frage, inwiefern es sich bei Exhibitionismus um eine „abnormale“ bzw. „pathologische“ sexuelle Betätigung handele. Der Jurist Prof. Dr. Helmut Weber etwa ordnete 1940 Exhibitionismus den "Sexualpathologien“ zu und kam zu dem Schluss: „Unter den Kinderschändern bilden sie eine kleine, aber doch bedeutsame Gruppe“. ${ }^{34}$ Hans Koopmann, Obermedizinalrat und als Gerichtsarzt tätig, sah Exhibitionismus zudem als „Großstadtphänomen“ an und hielt eine Vererbbarkeit desselben für möglich. ${ }^{35} \mathrm{Im}$ folgenden Fall folgte das Berliner Landgericht der These von Weber: Walter H., ein wegen Exhibitionismus vorbestrafter Stadtobersekretär, war im März 1943 von mehreren Müttern bei der Polizei angezeigt worden und wurde im April auf Basis des $\S 176$ Abs. 3 StGB zu zwei Jahren Zuchthaus verurteilt. ${ }^{36}$ Das Gericht stufte ihn in der Urteilsbegründung als "stark triebhafte[n] Mensch[en] [...] mit ausgesprochen exhibitionistischen Neigungen" ein. Laut Anklage hatte sich Walter H. im Februar und März 1943 mehrfach vor kleinen Mädchen entblößt und anschließend uriniert und/oder onaniert. Zwar wäre auch bei einer Verurteilung nach $\S 183$ eine Freiheitsstrafe von zwei Jahren Gefängnis möglich gewesen, allerdings kam selten das Höchstmaß zur Anwendung. Zudem unterschieden sich die Haftbedingungen in den Gefängnissen und Zuchthäusern zu diesem Zeitpunkt erheblich.

Verglichen mit der Verurteilung von Walter H. erhielt der ebenfalls nach $\S 176$ Abs. 3 StGB verurteilte Bühnenarbeiter Dietrich G. im Oktober 1943 mit neun Monaten Gefängnis eine recht geringe Strafe - obwohl der ihm zur Last gelegte Missbrauch der fünfährigen Rita schwerwiegend erscheint. ${ }^{37} \mathrm{Ihm}$ wurde vorgeworfen, das Mädchen im Juli 1943 gemeinsam mit der etwa gleichaltrigen Ingrid auf eine Bootsfahrt eingeladen, anschließend das Boot ins Schilf gefahren, sein erregtes Glied herausgeholt und sich zum Vollzug „beischlafähnlicher Bewegungen“ auf Rita gelegt zu haben. In der Urteilsbegründung wird auf sein recht junges Alter (zum Tatzeitpunkt war G. 18 Jahre alt) und eine nicht vorhandene "Neigung" rekurriert. So heißt es in der Anklageschrift, bei Dietrich G. lasse sich „eine verbrecherische Neigung nicht feststellen“, vielmehr sei seine Tat „als eine Pubertätsentgleisung aufzufassen“.

34 Helmut Weber, Die Bestrafung des Exhibitionismus, in: Monatsschrift für Kriminalpsychologie und Strafrechtsreform 31(12), 1940, 273-281. Nach Eghigian erklärt die zunehmende Verurteilung von Exhibitionisten nach 176/3 auch den Anstieg der Verurteilungen gemäß des Sexualstrafrechts zwischen den Jahren 1933 und 1936 von mehr als 15.000 auf mehr als 19.0oo Fälle, vgl. Eghigian, The Corrigible, 43 und 220.

Hans Koopmann, Exhibitionismus, in: Monatsschrift für Kriminalpsychologie und Strafrechtsreform 33(1/2), 1942, 18-26.

36 Alle folgenden Zitate aus Fallakte Walter H., LAB, 358-o2, Nr. 104114.

37 Fallakte Dietrich G., LAB, 358-o2, Nr. 103685. 
Der Typus ,jugendlicher Täter“ taucht bereits als Kategorie bei Richard von Krafft-Ebing auf, der im 19. Jahrhundert den Begriff der „Pädophilie“ geprägt hatte, und war ein zentraler Faktor bei der Beurteilung, ob die vorgeworfene Tat als eine "Neigungstat" oder eine "Gelegenheitstat" einzuordnen sei. ${ }^{38}$ Damit einher gingen zeitgenössische Vorstellungen von männlicher Sexualität, wonach diese grundsätzlich als triebhaft und mitunter schwer zu steuern galt. ${ }^{39}$ Dies impliziert indes auch in der Zeit des Nationalsozialismus nicht, dass Frauen sexuelle Bedürfnisse abgesprochen wurden. So heißt es in dem im Januar 1942 gefällten Urteil gegen die Buchhalterin Frieda E., der vorgeworfen wurde, ihren Pflegesohn Friedrich ab einem Alter von 13 Jahren jahrelang sexuell missbraucht zu haben: „[M]it Rücksicht auf das Geständnis und mit Rücksicht darauf, dass sie in sexueller Beziehung von ihrem Ehemann in keiner Weise befriedigt worden ist, sind ihr mildernde Umstände zugebilligt worden. ${ }^{40}$ Frieda E. wurde zu zwei Jahren Gefängnis verurteilt und nach eineinhalb Jahren begnadigt. Die Gerichtsakte von Frieda E. enthält eine weitere spannende Quelle, die auf vorherrschende Geschlechterbilder verweist: So berichtete das Frauengefängnis Leipzig in einer Beurteilung der Gefangenen aus dem Jahr 1942, ,wieweit sie vielleicht das Leben des damals jungen Menschen verdorben hat, dessen ist sie sich wohl nicht endgültig bewusst, zumal sie keine eigenen Kinder hat". ${ }^{41}$ Aufgrund der Tatsache, dass die zu diesem Zeitpunkt 42-jährige Frieda E. keine (biologische) Mutter war, wurde ihr abgesprochen, das Ausmaß und die Folgen der ihr vorgeworfenen Taten zu erkennen.

Obwohl die hier skizzierten drei Urteile sämtlich zur Zeit des Zweiten Weltkrieges gefällt wurden, als sogar die Verhängung von Todesurteilen bei einer Bestrafung nach den $\S \S 176-178$ StGB möglich geworden war, urteilten die Richter vergleichsweise milde, ${ }^{42}$ wenn sie die Taten als situativ einordneten, wie im Fall von Dietrich G., oder auf andere „externe Faktoren“,

$38 \quad$ Richard von Krafft-Ebing, Unzucht mit Individuen unter 14 Jahren, in: ders., Psychopathia Sexualis. 13. Aufl. Stuttgart 1912 [1. Aufl. 1886], 413-421, hier 414.

39 Vgl. dazu auch das Beispiel des im Juni 1943 vor dem Landgericht verurteilten Hans H., bei dem der ihm zur Last gelegte Missbrauch von einem zwölfährigen Mädchen auf eine mangelnde sexuelle Befriedigung durch die Ehefrau zurückgeführt wurde, Fallakte Hans H., LAB, 358-o2, Nr. 104035; Lieske, Maßnahmenstaat, 197-198.

40 Fallakte Frieda E., LAB, 258-o2, Nr. 103738.

41 Ebd.

42 Besonders deutlich wird dies vor dem Hintergrund der im Zweiten Weltkrieg gefällten Urteile der Sondergerichte. So war es nach Kriegsbeginn keine Seltenheit, dass Personen wegen Delikten wie dem „illegalen Abhören ausländischer Radiosender“ oder gegen das Regime gerichteten Aussagen zu jahrlangen Haftstrafen verurteilt wurden. Nicht selten kam es auch zu Todesurteilen wegen „Plünderung“ oder „Diebstahl“. 
wie eine defizitäre Sexualität in der Ehe zurückführten, wie bei Frieda E. Ging das Gericht aber wie bei Walter H. von einer den Taten zugrunde liegenden „Neigung" aus, war nicht nur das Strafmaß höher, vielmehr führte dies häufig zur Anordnung weiterer Maßnahmen: Der 26-jährige Paul H. etwa wurde im Dezember 1937 wegen „Notzucht“(Vergewaltigung) in einem Fall und sechsfach versuchter „Notzucht“, begangen an erwachsenen Frauen, sowie „unzüchtigen Handlungen“ an einem zehnjährigen Mädchen zu einer Zuchthausstrafe von insgesamt acht Jahren verurteilt. Zusätzlich ordnete das Landgericht seine „Entmannung“ sowie die Unterbringung in einer Heil- und Pflegeanstalt an. ${ }^{43}$ Letzteres begründete das Gericht mit einer möglichen „Erfolglosigkeit“ der Kastration: „Für diesen Fall wäre es für die Allgemeinheit unerträglich, dass der Angeklagte nach Verbüßung seiner 8-jährigen Zuchthausstrafe im besten Mannesalter von 34 Jahren wiederum Gelegenheit erhält, sich als Sittlichkeitsverbrecher zu betätigen." Hier zeigt sich ein weiterer Diskursstrang innerhalb der nationalsozialistischen Kriminalpolitik: Galt den einen die (Zwangs-) Kastration von Sexualstraftätern als (präventives) Heilmittel, zweifelten andere den „Erfolg“ dieser Maßnahme an, so dass häufig neben dem körperlichen Eingriff eine Internierung erfolgte. Dabei wurden die Experten nicht müde zu betonen, dass die Kastration nicht als Bestrafung, sondern vielmehr als „zusätzliche Sicherungsmaßregel“ anzusehen sei. ${ }^{44}$

\section{Der Blick auf die Betroffenen}

Die Gerichtsakten erlauben meist keine Einbeziehung der subjektiven Sicht der Betroffenen auf das Geschehene. Diese bleiben in den Quellen auf ihre Rolle als Zeug:innen beschränkt. Verschiedene Autor:innen haben anhand von Strafprozessen im Kaiserreich und der Weimarer Republik nachgewiesen, dass diese Rolle schon vor der Zeit des Nationalsozialismus häufig insofern mit einer Abwertung der Betroffenen einherging, als sie wegen ihres Alters und/oder

\footnotetext{
43 Alle folgenden Zitate aus Fallakte Paul H., LAB, 358-o2, Nr. 22708, vgl. dazu auch Lieske, „Gemeingefährlichen“, 415 .

44 Vgl. z.B. Franz Rattenhuber, Der gefährliche Sittlichkeitsverbrecher. Bottrop 1939, 17. Zur zeitgenössischen Debatte um die Kastrationen auch Marc Dupont, Sexualwissenschaft im „Dritten Reich“. Eine Inhaltsanalyse medizinischer Zeitschriften. Frankfurt a. M. 1996, 101-132; Susanne zur Nieden, „Entmannung“. Zum juristisch-medizinischen Umgang mit abweichendem Sexualverhalten im Nationalsozialismus, in: Zeitschrift für Geschichtswissenschaft 53(9), 2005, 791-808.
} 
ihrer sozialen Herkunft als unglaubwürdig galten..$^{45}$ Auch wenn die Expertise von geschulten Begutachter:innen der Zeug:innen vor Gericht im Nationalsozialismus an Bedeutung verlor, ${ }^{46}$ wurden die Betroffenen während der Ermittlungen auf ihre Glaubwürdigkeit hin überprüft. Die Polizei fragte dazu in der Regel zunächst bei den entsprechenden Jugendämtern sowie manchmal auch den Lehrer:innen an und informierte sich über die sozialen und häuslichen Verhältnisse, in denen die Kinder und Jugendlichen aufwuchsen.

Vor Gericht wurden Jungen und Mädchen als Zeugen durchaus unterschiedlich wahrgenommen: Während Erstere tendenziell als zuverlässiger galten, erschienen Mädchen häufiger als (unzuverlässige) Zeuginnen, insbesondere dann, wenn sie als "sexuell verwahrlost" eingestuft wurden. ${ }^{47} \mathrm{Ob}$ diese Differenz Auswirkungen auf die Rechtsprechung hatte, wäre noch zu prüfen. So gibt es in der NS-Zeit durchaus Beispiele dafür, dass betroffene Mädchen zwar abgewertet, aber dennoch als glaubwürdig eingestuft wurden, vermutlich, um eine Verurteilung zu erwirken. ${ }^{48}$

In den Akten finden sich Hinweise darauf, wie belastend die erfahrene Gewalt selbst und die Aussagen vor Polizei und Gericht waren. Erna G., die als Kind jahrelang von ihrem Vater missbraucht worden war, wandte sich 1942 im Alter von 19 Jahren an die Kriminalpolizei. Auf die Frage, warum sie nicht früher gekommen sei, antwortete sie: „Ich habe mich immer nicht getraut, meiner Mutter das zu erzählen. Vater hat auch ständig gedroht, dass er sich dann das Leben nehmen würde und ich käme ins Zuchthaus.“49 Der in der Akte dokumentierte Versuch des Vaters, sich und Ernas Mutter bei der Festnahme mit einem Dolch das Leben zu nehmen, legt nahe, dass die Sorgen

45 Peter Dudek, „Liebevolle Züchtigung“. Ein Mißbrauch der Autorität im Namen der Reformpädagogik. Bad Heilbrunn 2012; Brigitte Kerchner, „Kinderlügen“? Zur Kulturgeschichte des sexuellen Missbrauchs, in: Ute Finger-Trescher/Heinz Krebs (Hrsg.), Mißhandlung, Vernachlässigung und sexuelle Gewalt in Erziehungsverhältnissen. Gießen 200o, 15-41; dies., ,Unbescholtene Bürger" und "gefährliche Mädchen“ um die Jahrhundertwende. Was der Fall Sternberg für die aktuelle Debatte um sexuellen Missbrauch an Kindern bedeutet, in: Historische Anthropologie 6(1), 1998, 1-32; Heather Wolffram, Forensic Psychology in Germany. Witnessing Crime, 1880-1939. Canterbury 2018.

46 Ebd., 224.

47 Ebd., 209-211; Kerchner, Körperpolitik, 274.

48 Vgl. dazu das an anderer Stelle ausgeführte Beispiel von zwei Mädchen, deren Glaubwürdigkeit sowohl der Angeklagte als auch dessen Rechtsanwalt wiederholt aufgrund ihrer angeblichen "Verwahrlosung“ anzweifelten. Obgleich das Gericht dieser Einschätzung folgte, stufte es die Mädchen als glaubwürdig ein und verurteilte den Angeklagten und seinen Freund auf Basis ihrer Aussagen zu Haftstrafen wegen "Unzucht“ an Minderjährigen, Fallakte Valentin W. und Emil H., LAB 358-02, Nr. 51612, vgl. Lieske, „Gemeingefährlichen“, 421-422.

Fallakte Johann G., LAB, 358-02, Nr. 104085 . 
seiner Tochter nicht unbegründet waren. Erna G. musste zudem im Rahmen der Ermittlungen mit einer Anzeige wegen "Blutschande“ nach $§ 173$ StGB $^{50}$ rechnen, da sie angab, ihr Vater habe mit ihr den "Geschlechtsakt" auch noch vollzogen, als sie bereits älter als 18 Jahre war. Fachleute und Gerichte gaben gerade im Fall von „Blutschande“/,Inzest“ den betroffenen Mädchen häufig mindestens eine Mitschuld an den Taten. ${ }^{51}$ So stellte der Mediziner Georg Schwab, Mitarbeiter an der kriminalbiologischen Forschungsstelle im Zuchthaus Ludwigsburg, 1938 in einem Artikel in der Monatsschrift für Kriminalbiologie und Strafrechtsreform Behauptungen über "mitschuldige Töchter“ und "geschlechtlich hemmungslose Mädchen“ auf, die nur in wenigen Fällen Widerstand gegen den Missbrauch durch ihre Väter geleistet hätten. Schwab unterstellte sogar ein "mehr oder minder bewusstes Liebesspiel der Mädchen“ oder auch ein „dirnenhaftes Sichanbieten der sittenlosen Tochter“. ${ }^{52}$

\section{Ausblick und Diskussion}

Wird der Fokus auf die nach 1933 eingeführten Maßnahmen wie Zwangskastrationen oder die Einweisung von vorbestraften "Kinderschändern“ in Konzentrationslager gelegt, lässt sich konstatieren, dass eine Verurteilung wegen sexuellem Kindesmissbrauch im Nationalsozialismus tatsächlich härtere Konsequenzen haben konnte als beispielsweise in der Weimarer Republik. Daraus nun jedoch abzuleiten, dass das Regime etwa den Schutz von Kindern ernster genommen hätte als demokratische Systeme vor und nach der Zeit des Nationalsozialismus, leitet fehl. Denn den harten Urteilen und Repressionsmaßnahmen gegen Einzelne stehen zahlreiche Beispiele für eine Bagatellisierung der Taten gegenüber, auch wenn das im Urteil zur Sprache kommende Ausmaß der verübten sexuellen Gewalt gravierend war und/oder sich - und dies mag überraschen - auf gleichgeschlechtliche Handlungen

$50 \quad$ In Deutschland stellt der $\S 173$ seit der Einführung des Reichsstrafgesetzbuches Vaginalverkehr zwischen (leiblichen sowie bis 1973 auch verschwägerten) Verwandten unter Strafe, wobei die Strafbarkeit von Geschlechtsverkehr unter Geschwistern an das Alter (mindestens 18 Jahre) gekoppelt ist. Seit einigen Jahren wird indes über eine grundlegende Reform des $§ 173$ debattiert, so hat der Ethikrat 2014 für die Straffreiheit von „Beischlaf" unter Geschwistern plädiert, die nicht zusammen aufgewachsen sind.

51 Die These einer „Mitschuld“ der betroffenen Mädchen an „Inzest“ findet sich bereits im Kaiserreich, vgl. dazu Heike Schmidt, Gefährliche und gefährdete Mädchen. Weibliche Devianz und die Anfänge der Zwangs- und Fürsorgeerziehung. Opladen 2002.

$5^{2}$ Dr. med. G. Schwab, Zur Biologie des Inzests, in: Monatsschrift für Kriminalbiologie und Strafrechtsreform 29(6), 1938, 257-276, hier 268 und 273 . 
bezog: Nicht jeder Mann, der einen Jungen sexuell missbrauchte, galt den Nationalsozialisten als „homosexuell“. 53

Es scheint somit, als wäre die Grenze weniger zwischen „gleichgeschlechtlich“ oder "heterosexuell" verlaufen, als vielmehr zwischen der Einordnung einer Tat als „Neigungstat“ oder „Ersatzhandlung“. Männer, denen eine „homosexuelle Neigung" zugesprochen wurde, waren genauso wie Männer, die als „pädophil“ oder „Exhibitionisten“ im Sinne einer sexuellen „Neigung“ galten, tatsächlich von härteren Urteilen und Maßnahmen bedroht. „Ersatztaten“ führten Mediziner, Polizist:innen und Juristen demgegenüber auf Faktoren wie eine mangelnde oder unbefriedigende Sexualität in der Ehe, auf (altersbedingte) männliche sexuelle Instinkte oder ein angeblich (falsches) Verhalten des bzw. der Betroffenen selbst zurück. Entscheidend für die Urteile war somit - und dies ist typisch für die nationalsozialistische Rechtspraxis - die Zentrierung auf die Täter:innenpersönlichkeit und weniger die vorgeworfene Tat als solche.

Die Unterscheidung zwischen „Neigungstaten“ und „Ersatzhandlungen“ ist jedoch kein Spezifikum nationalsozialistischer Kriminalpolitik. Sowohl vor 1933 als auch in der heutigen Sexualwissenschaft spielt sie bei der Erklärung für sexuelle Gewalt eine große Rolle. Die Besonderheit liegt vielmehr in der Rechtspraxis und der Implementierung zusätzlicher Maßnahmen wie der Zwangssterilisation. Der nationalsozialistische Staat ermöglichte im kriminalund biopolitischen Bereich ein hohes Maß an Repression und Willkür, das sich mit voller Wucht auch gegen Menschen richten konnte, die wegen Sexualstraftaten verurteilt und pathologisiert wurden.

Die besondere Verletzlichkeit der betroffenen Kinder und Jugendlichen stand dabei indes nicht im Vordergrund. Mehr noch: Nicht selten erfuhren auch die betroffenen Kinder und Jugendlichen infolge der erfahrenen sexuellen Gewalt weitere Repressionen. So mussten Mädchen beispielsweise mit Zwangssterilisationen infolge von Missbrauch rechnen. Jugend allgemein und deren Sexualität im Speziellen waren zentral für die Konzeption der "Volksgemeinschaft", in der jegliche Form von "Verwahrlosung“ (etwa durch frühzeitig erlebte Sexualität, auch in Folge eines Missbrauchs!) als Gefahr für die gesamte Gesellschaft galt. ${ }^{54}$

53 Vgl. das an anderer Stelle von mir ausgeführte Beispiel von Heinz G., der 1944 vor Gericht stand, weil ihm vorgeworfen wurde, einen fünfährigen Jungen sexuell missbraucht zu haben. G. galt dem Gericht nicht als Homosexueller, sondern als „Gelegenheitstäter“, Fallakte Heinz G., LAB A Rep. 358-o2, Nr. 104010, vgl. Lieske, Maßnahmenstaat, 196.

54 Vgl. zum Umgang mit der Sexualität von Jugendlichen im Nationalsozialismus Robert G. Waite, Teenage Sexuality in Nazi Germany, in: Journal of the History of Sexuality 8(3), 1998, 434-467. 
Stigmatisierende Diskurse über Betroffene endeten aber nicht mit dem Ende der nationalsozialistischen Herrschaft, ${ }^{55}$ ebensowenig wie kriminalpolitische Konzepte obsolet sind, in denen suggeriert wird, ein gesellschaftlicher Ausschluss von (potentiellen) Täter:innen in Form eines „Wegsperrens“ würde sexuellen Kindesmissbrauch grundsätzlich verhindern. Gerade die Auseinandersetzung mit der Geschichte des Nationalsozialismus bietet hier ein hilfreiches Instrument zur Analyse aktueller Diskurse. Künftige Forschungsprojekte sollten deshalb verstärkt Kontinuitäten im Hinblick auf die Entwicklung und Struktur von Institutionen, wie etwa Schulen, Heimen oder auch der Kirche in den Blick nehmen, die im Bereich der sexuellen Gewalt gegen Kinder und Jugendliche nach wie vor (leider) eine zentrale Rolle spielen. ${ }^{56}$

55 Vgl. zu Kontinuitäten geschlechtsspezifischer Zuschreibungen bei den Betroffenen bis in die 196oer und 1970er Jahre auch Jan-Henrik Friedrichs, Delinquenz, Geschlecht und die Grenzen des Sagbaren. Sexualwissenschaftliche Diskursstränge zur Pädophilie, 196o1995, in: Zeitschrift für Sexualforschung 30(2), 2017, 161-182.

56 Hinweise darauf liefert z.B. Claus Koch, Das Kind als Feind, das Kind als Freund. Was haben national-sozialistisches Erziehungserbe und pädophile Ideologie mit der gegenwärtigen Missbrauchsdebatte zu tun?, in: Sabine Andresen/Wilhelm Heitmeyer (Hrsg.), Zerstörerische Vorgänge. Missachtung und sexuelle Gewalt gegen Kinder und Jugendliche in Institutionen. Weinheim/Basel 2012, 228-242. 